\title{
¿ES EFICAZ LA CONFIGURACIÓN ACTUAL DE CONSTITUCIÓN DE LA REPRESENTACIÓN SINDICAL DE LOS TRABAJADORES EN LAS EMPRESAS DISPERSAS? ${ }^{1}$
}

\author{
David Gutiérrez Colominas \\ Investigador Postdoctoral \\ Universidad Autónoma de Barcelona
}

\begin{abstract}
La constitución de la representación sindical de los trabajadores en las empresas dispersas presenta se encuentra en un escenario de cambios, propiciados por la irrupción de nuevas formas de organización empresarial basadas en la economía colaborativa. La desaparición de la realidad física del centro de trabajo, que es la tónica imperante en la actualidad en las empresas dispersas, plantea distintos problemas jurídicos en las relaciones colectivas, derivados esencialmente de la falta de actualización del marco jurídico regulador del concepto "centro de trabajo", pero, a su vez, ofrece un escenario excepcional para el fomento de la participación sindical en este tipo de empresas. Así, este artículo aborda si el actual marco regulador ofrece respuestas eficaces para garantizar la efectiva constitución de la representación sindical y cómo influye este fenómeno en la participación de los sindicatos en las relaciones laborales. Para ello, el presente artículo se centra en la caracterización legal e interpretación jurisprudencial del concepto "centro de trabajo", introduciendo además distintas propuestas de mejora de la regulación vigente, a fin de garantizar el ejercicio del derecho de los trabajadores a participar en la empresa.
\end{abstract}

The constitution of the union representation of workers in dispersed companies is in a scenario of changes, propitiated by the irruption of new forms of business organization based on the collaborative economy. The disappearance of the physical reality of the workplace, which is the prevailing trend in dispersed companies today, exposes different legal problems in collective relations, essentially derived from an outdated legal regulation of the concept of "workplace". But, at the same time, it offers an exceptional scenario for the promotion of trade union participation in this type of companies. Thus, this article deals with whether the current regulatory framework offers effective responses to guarantee the effective constitution of trade union representation, and how this phenomenon influences the participation of trade unions in

\footnotetext{
${ }^{1}$ El presente trabajo se ha elaborado en el marco del Proyecto de I+D+I, del Programa estatal de investigación, desarrollo e innovación orientada a los retos de la sociedad, con el título "La representación laboral en las empresas dispersas y en red: problemática, disfunciones y propuestas correctoras" (DER2017-83189-R), IP: Xavier Solà Monells y Ricardo Esteban Legarreta.
} 
labour relations. To this end, this article focuses on the legal characterization and jurisprudential interpretation of the concept of "workplace", also introducing various proposals for improving current regulations, in order to guarantee the exercise of the right of workers to participate in the company.

IUSLabor 1/2019, ISSN 1699-2938, p. 287-306

DOI. 10.31009/IUSLabor.2019.i01.08

Title: Is the current configuration of the constitution of the union representation of workers in dispersed companies effective?

Palabras clave: representación sindical, ámbito de constitución, empresas dispersas, microcentros de trabajo.

Keywords: trade union representation; scope of constitution; dispersed enterprises; work microcentres.

\section{Sumario}

1. La representación de los trabajadores y la economía colaborativa: ¿Viejas fórmulas para nuevos problemas?

2. Una visión histórica sobre el estado de la cuestión

3. La caracterización del centro de trabajo en las empresas dispersas

4. Algunas medidas para mejorar la eficacia de la constitución de la representación sindical en empresas dispersas 


\section{La representación de los trabajadores y la economía colaborativa: ¿Viejas fórmulas para nuevos problemas?}

Las tecnologías de la información están cambiando el mundo del trabajo. La irrupción de Internet en el mercado ha modificado la forma en la que vivimos en sociedad, e incluso, ha supuesto la creación de nuevas formas de organización empresarial. En efecto, la llamada "economía colaborativa", junto con otros fenómenos paralelos ${ }^{2}$, ha revolucionado las relaciones laborales, mediante la introducción de aplicaciones informáticas que se encargan de distribuir las tareas a un grupo homogéneo de personas, cuya calificación jurídica como trabajadores es más que discutible como consecuencia de la falta de actualización normativa ${ }^{3}$. De esta manera, las empresas de la economía colaborativa han originado una descentralización productiva extrema, que requiere una urgente reforma del marco jurídico a propósito de la intervención y límites de las nuevas tecnologías en el desarrollo de actividades económicas.

Ante este escenario, la creación de plataformas virtuales, que actúan como posibles empleadores, ha puesto de relieve la intervención de nuevas formas de organización empresarial en el denominado mercado único digital ${ }^{4}$, que utilizan las tecnologías de la información para desarrollar su actividad económica y ser más competitivas. Las empresas digitales son precisamente un actor relativamente nuevo, que son concebidas para operar exclusivamente a través de Internet, y su actividad económica fundamental se basa en la intermediación o la venta directa online ${ }^{5}$.

\footnotetext{
${ }^{2}$ Tal y como señala PÁramo Montero, P., "Las nuevas formas emergentes de trabajo. Especial referencia a la economía colaborativa", Revista del Ministerio de Empleo y Seguridad Social, vol. 128, 2017, p. 184, la "gig economy" evoluciona en paralelo con otros fenómenos fruto de la irrupción de la tecnología en la vida en sociedad, como por ejemplo las "smart cities" o el "smart working", entre otros.

${ }^{3}$ Sobre esta cuestión, Todolí SIGNES, A., "La regulación especial del trabajo en la Gig Economy", Redes, vol. 15,2017 , p. 71 apunta a la masiva inaplicación de las protecciones concedidas por el Derecho del Trabajo, permitiendo su calificación como autónomos, cuestión esta que cristaliza el fenómeno de la huida del Derecho del trabajo constatado de forma visionaria por BAYLOS GRAU, A., "La «huída» del Derecho del trabajo. Tendencias y límites de la deslaboralización.", en El trabajo ante el cambio de siglo : un tratamiento multidisciplinar, Marcial Pons, Barcelona, 2000, p. 35-54.

${ }^{4}$ Esta denominación fue adoptada por la Comisión Europea en la Comunicación de la Comisión al Parlamento Europeo, al Consejo, al Comité Económico y Social Europeo y al Comité de las Regiones, "Una Estrategia para el Mercado Único Digital de Europa" [COM(2015)192], y engloba una serie de actuaciones que pretenden una utilización más eficaz de las oportunidades que ofrecen las nuevas tecnologías, e integra iniciativas que abarcan distintos ámbitos de actuación, como por ejemplo la regulación de las telecomunicaciones, de derechos de propiedad intelectual y de legislación sobre protección de datos, de gestión de las ondas de radio y de aplicación del Derecho de la competencia.

${ }^{5}$ Vid. Padilla Meléndez, A.; Serarols I Tarrés, C., "Las caractertiscas del empresario y el éxito de las empresas puramente digitales", Información Comercial Española, ICE: Revista de economía, vol. 833, 2006, p. 156.
} 
Ahora bien, el marco jurídico europeo y nacional no ha sido capaz de dar una respuesta a estos nuevos actores, que operan en una realidad normativa concebida antes de la irrupción de las tecnologías de la información en el mercado de trabajo. El marco jurídico que regula las relaciones laborales ha permanecido prácticamente impasible ante la irrupción de estas nuevas circunstancias económicas. Concretamente, el Real Decreto Legislativo 2/2015, de 23 de octubre, por el que se aprueba el texto refundido de la Ley del Estatuto de los Trabajadores (en adelante, TRLET), permanece prácticamente intacto desde su redacción en el hoy derogado Real Decreto Legislativo 1/1995, de 24 de marzo, por el que se aprobó el texto refundido de la Ley del Estatuto de los Trabajadores.

Sin embargo, la cuestión no es tanto la falta de reacción jurídica del legislador, sino cómo afecta de forma más intensa a ciertas parcelas de las relaciones laborales. Y una de ellas es la participación de la representación de los trabajadores en la empresa, canalizada jurídicamente mediante el derecho a la información, consulta y participación en la empresa ex artículo 4 TRLET.

El vigente modelo español de representación colectiva de los trabajadores es un modelo de doble canal representativo ${ }^{6}$, o dual ${ }^{7}$, en el que coexisten de forma autónoma dos figuras: la representación sindical, formado por las secciones sindicales y los delegados sindicales; y la representación unitaria, compuesta por los comités de empresa y los delegados de personal. En la configuración de la constitución de ambas figuras existe una circunstancia que es especialmente relevante con relación a la descentralización que ha generado la irrupción de las empresas de la economía colaborativa: la consideración del centro de trabajo como unidad de referencia para la constitución de la representación de los trabajadores. Tanto el artículo 62 y 63.a del TRLET, como el artículo 10 de la Ley Orgánica 11/1985, de 2 de agosto, de Libertad Sindical (en adelante, LOLS) identifican el centro de trabajo como unidad electoral básica, si bien en el caso del artículo 10 de la LOLS la jurisprudencia ha sido más permisiva con el ámbito de constitución de las secciones sindicales ${ }^{8}$.

\footnotetext{
${ }^{6}$ CASAS BAAmonde, M. E. y Escudero RodríGuez, R., "Representación sindical en el sistema español de relaciones laborales", Revista española de Derecho del Trabajo, vol. 17, 1984, p. 51-92.

${ }^{7}$ Véase la STS de 31 de enero de 2001 (Rec. 1959/2000), F.D. V.

${ }^{8}$ Véase la STS de 18 de julio de 2014 (Rec. 91/2013), que tomando en consideración las STC 84/1989 de 10 de mayo, 75/1992 de 14 de mayo, 201/1999 de 8 de noviembre, 132/2000 de 16 de mayo, así como las STS de 15 de julio de 1996 (Rec. 3432/1995) y 26 de junio de 2008 (Rec. 18/2007), sostuvo que la opción que se ofrece en el artículo 10.1 de la LOLS entre nombrar los Delegados Sindicales a nivel de empresa o de centro de trabajo pertenece al sindicato en cuestión como titular del derecho de libertad sindical
} 
Ante este escenario, anclado a una visión antigua de las relaciones laborales, la hipótesis de partida de este trabajo es que la dinámica de funcionamiento inherente a la economía colaborativa, fundamentado en trabajadores dispersos sin centros de trabajo stricto sensu, favorece un modelo de representación de los trabajadores más fundamentado en la representación sindical que unitaria, dada la escasa flexibilidad jurisprudencial que ha mostrado la segunda de ellas. Esto puede suponer un incremento de la constitución e implementación de los sindicatos en las relaciones laborales, y, por lo tanto, un aumento del ejercicio del derecho fundamental a la libertad sindical (artículo 28.1 de la Constitución Española, en adelante, CE). Sin embargo, el marco jurídico debe ser actualizado para adaptar el ejercicio del derecho a la participación de los trabajadores en las empresas dispersas. De hecho, desde un punto de vista sociológico, algunos estudios ya han indicado el reducido margen de representación que ostentan un elevado número de empresas con menos de 6 trabajadores en plantilla, como consecuencia del escaso tratamiento de fenómenos como la descentralización productiva ${ }^{9}$.

Y es en este punto donde nace la pretensión de esta contribución, que pretende examinar el ámbito de constitución de las secciones sindicales y la eficacia de la regulación actual en el marco de las empresas dispersas. Partiendo de la hipótesis del surgimiento de nuevas formas de economía en un mercado digitalizado, esta contribución abordará si el actual marco jurídico garantiza la participación de los trabajadores en la empresa, centrándose exclusivamente en la dimensión sindical. Para ello, se efectuará un breve repaso sobre el estado de la cuestión en la jurisprudencia, la problemática que presenta el concepto de centro de trabajo en las empresas dispersas como unidad de adscripción territorial para la constitución de secciones sindicales, y, por último, la formulación de una serie de propuestas de mejora del marco legal para la constitución efectiva de la representación sindical en empresas dispersas.

\section{Una visión histórica sobre el estado de la cuestión}

Cómo ya se ha apuntado, la unidad electoral de referencia para la elección de representantes sindicales de los trabajadores se ha mantenido legalmente impasible a lo largo de los años. El contenido de la LOLS, aprobada en 1985, no ha sido modificado en ninguna ocasión, a pesar de haberse producido múltiples reformas laborales ${ }^{10}$. Si

\footnotetext{
${ }^{9}$ En este sentido, véase Alós, R., BEnEYTO, P. J., JÓDAR, P., MOLINA, O. y VIDAL, S., La representación sindical en España, Fundación $1^{\circ}$ de Mayo, 2015.

${ }^{10} \mathrm{Si}$ bien es cierto que algunas de esas reformas han retocado aspectos de la representación unitaria (1994 y 2010), la unidad electoral de referencia para la elección de representantes unitarios y sindicales de los trabajadores no ha sido objeto de modificación. Esta circunstancia ha sido interpretada por la doctrina como un indicio de prueba de la obsolescencia del modelo vigente por la falta de adaptación a la compleja realidad empresarial. Véase, en este sentido, VAldÉs DAL-RE, F. y Molero MARAÑON, L., "Presentación", en La representación de los trabajadores en las nuevas organizaciones de empresa,
} 
bien esta circunstancia evidencia la nula voluntad del legislador de ampliar el derecho fundamental a la libertad sindical, la jurisprudencia ha venido a ampliar la interpretación de este derecho fundamental, ofreciendo una necesaria flexibilidad que debería trasladarse al contenido de la norma.

La evolución jurisprudencial sobre cómo debía interpretarse el artículo 10.1 de la LOLS, que es el que constata el centro de trabajo y la empresa como unidad electoral de referencia a efectos de constituir la representación sindical, sufrió distintos giros hasta alcanzar la doctrina, hoy vigente, contenida en la sentencia del Tribunal Supremo de 18 de julio de 2014 (Rec. 91/2013). En un momento inicial, la sentencia del Tribunal Supremo de 15 de julio de 1996 (Rec. 3432/1995) sostuvo que la decisión de constituir la sección sindical tomando como ámbito de referencia electoral la empresa o el centro de trabajo corresponde al sindicato, ya que el artículo 8.1 de la LOLS, al recoger el catálogo de potestades de los afiliados a un sindicato constata dos opciones (empresa o centro de trabajo) cuya elección corresponde a los afiliados. El Tribunal trasladó el razonamiento del artículo 8 de la LOLS a la interpretación del artículo 10.1 de la LOLS, expandiendo los límites de la libertad de organización interna de los sindicatos y permitiendo la autoelección del censo electoral que más le convenga.

No obstante, la sentencia del Tribunal Supremo de 10 de noviembre de 1998 (Rec. 2123/1998) modificó la interpretación del artículo 10.1 de la LOLS, sosteniendo que debe existir una necesaria correlación entre la representación unitaria y la representación sindical de los trabajadores. En particular, señaló que la posibilidad de acudir a la empresa o al centro de trabajo no es una decisión que corresponde al sindicato de forma libre, sino que dependerá de cómo se hayan constituido los órganos de representación unitaria de los trabajadores. En términos prácticos, la Sala sostuvo que la unidad de referencia electoral debe ser coincidente entre la representación unitaria y representación sindical, ya que ambas deben mantener una cierta coherencia en los criterios y modos de participación en la empresa.

El último cambio interpretativo del artículo 10.1 de la LOLS fue introducido a raíz de la sentencia del Tribunal Supremo de 18 de julio de 2014 (Rec. 91/2013), que ha sido confirmada en diversas ocasiones $^{11}$, y se encuentra en vigor ${ }^{12}$. Este pronunciamiento

Ministerio de Trabajo e Inmigración, Madrid, 2010, p. 14-15 y SAÉZ LARA, C., "La representación colectiva de los trabajadores en la empresa", Revista del Ministerio de Trabajo y Asuntos Sociales, 58, 2005 , p. 315 y ss.

${ }^{11}$ Véase, por ejemplo, la Sentencia del Tribunal Supremo de 8 de febrero de 2018 (Rec. 274/2016), de 25 de enero de 2018 (Rec. 30/2017, de 6 de junio de 2017 (Rec. 216/2016), de 7 de marzo de 2017 (101/2016), de 3 de febrero de 2017 (Rec. 39/2016), 12 de julio de 2016 (Rec. 361/2014), entre muchas otras. 
judicial recupera la facultad de que el sindicato pueda seleccionar la unidad electoral de adscripción que considere oportuna, diferenciando la figura de la sección sindical con la del comité de empresa. En este sentido, el Tribunal efectúa un análisis que pone de relieve que la referencia para el Comité de empresa es el centro de trabajo, y sólo en ciertos casos se podrá constituir o un "comité de empresa conjunto", para posibilitar la representación unitaria cuando hay varios centros de trabajo pero de menos de 50 trabajadores (artículo 63.2 del TRLET ) o un "comité intercentros" para el supuesto contrario: muchos centros de trabajo con Comités a los que les puede interesar coordinarse (artículo 63.3 del TRLET ). En cambio, la referencia en el caso de las secciones sindicales es la empresa, y en segundo lugar, el centro de trabajo ex artículo 10.2 de la LOLS, matización que la Sala justifica en la aplicación práctica a sensu contrario: la escasa presencia de centros de trabajo de más de 250 trabajadores en el ordenamiento jurídico español supone que la exigencia de esa cifra para poder contar con Delegados Sindicales en relación a cada centro de trabajo equivaldría a dejar vacío de contenido el artículo 10.1 de la LOLS. De esta forma, los sindicatos tienen plena libertad para elegir la unidad electoral de referencia a efectos de constituir la representación sindical de los trabajadores en la empresa.

Esta posibilidad ha abierto un nuevo debate: ¿existe la posibilidad de constituir secciones sindicales en empresas o centros de trabajo con una plantilla inferior a 250 trabajadores? El quid de la cuestión radica en la aparente contradicción entre los artículos 8 y 10.1 de la LOLS, que facultan a los afiliados a un sindicato para la constitución de secciones sindicales sin ningún tipo de requisito a nivel de plantilla, pero que a posteriori parece condicionarse a la exigencia de una plantilla mínima. Esta discusión, que presenta algunos problemas prácticos ya resueltos ${ }^{13}$, ya fue abordada por el Tribunal Constitucional y el Tribunal Supremo ${ }^{14}$, que, en aras a potenciar el ejercicio del derecho a la libertad sindical, permitió la posibilidad de constituir secciones sindicales en cualquier empresa o centro de trabajo independientemente del número de

12 Para un estudio en profundidad, véase MIÑARRO YANINI, M. "La representación sindical se "independiza" de la unitaria: el crédito horario de los delegados sindicales (Comentario a la Sentencia del Tribunal Supremo, Sala 4. ${ }^{\text {, }}$, de 18 de julio de 2014, rec. no 91/2013)”, Revista de trabajo y Seguridad Social del Centro de Estudios Financieros, no 381, 2014, p. 163-167 y Moll NoGUERA, R., "Crédito de horas retribuidas de los delegados sindicales: (Sentencia del Tribunal Supremo de 18 de julio de 2014, RC 91/2013)", Revista de información laboral, no 11, 2014, p. 95-111.

${ }^{13}$ Así, por ejemplo, la sentencia del Tribunal Supremo de 11 de abril de 2001 (Rec. 1672/2000) abordó la problemática relativa a la disminución del número de trabajadores, dictaminando que la reducción de plantilla por debajo de la cifra de 250 trabajadores supone la pérdida del reconocimiento de los derechos previstos en el artículo 10.3 de la LOLS. No obstante, se mantendrá la condición de representante sindical ad intra.

${ }^{14}$ Véanse las sentencias del Tribunal Constitucional $n^{\circ}$ 4/1989 de 18 de enero, 75/1992 de 14 de mayo, 201/1999 de 2 de agosto, 132/2000 de 16 de mayo; y las sentencias del tribunal supremo de 12 de diciembre de 1989, 15 de julio de 1996 (Rec. 3432/1995) y 26 de junio de 2008 (Rec. 18/2007). 
personas trabajadoras que presenten servicios en la unidad de adscripción electoral correspondiente. De forma resumida, el Tribunal Constitucional ha señalado que el sentido de constatar un número determinado de plantilla tiene su razón de ser en el marco de las facultades previstas en el artículo 10 de la LOLS, pero dicha cifra no supone una limitación del derecho constitucional a la libertad sindical plasmado en el artículo 28.1 de la CE y desarrollado legalmente a través de la LOLS. El reconocimiento de la doble dimensión del derecho a la libertad sindical, a saber, la interna como expresión de autoorganización del Sindicato y externa como representantes del sindicato en cuestión, supuso la creación de dos categorías de delegados sindicales: los delegados sindicales ad intra y los delegados ad extra. La principal diferencia entre cada una de estas figuras radica en el haz de facultades legales reconocidas; mientras que los delegados sindicales ad intra actúan como meros representantes o portavoces de los afiliados, sin que tengan reconocidos ninguno de los derechos y garantías del artículo 10.3 de la LOLS, los delegados sindicales ad extra ostentarán la posibilidad de ejercer las facultades reconocidas en el artículo 10.3 de la LOLS. De esta forma, existirán dos tipos de delegados sindicales, los delegados sindicales ad intra (plantillas inferiores a 250 trabajadores, y con las facultades del artículo 10.3 LOLS limitadas) y los delegados sindicales ad extra (plantillas superiores a 250 trabajadores, con las posibilidades de ejercer los derechos previstos en el artículo 10.3 de la LOLS).

\section{La caracterización del centro de trabajo en las empresas dispersas}

Así pues, una vez examinada la interpretación del artículo 10 de la LOLS, conviene detenerse en una de las cuestiones principales de esta contribución, que no es otra que comprobar la eficacia del ámbito de constitución de la representación sindical en el marco de las empresas dispersas, y especialmente aquellas que tienen una estrecha conexión con las tecnologías de la información. Dentro de estas conviene efectuar una precisión no menor, relativa a la definición de centro de trabajo, que es el concepto que mayores problemas interpretativos presenta en la aplicación del artículo 10 de la LOLS: ¿son todos los centros de trabajo de las empresas dispersas centros de trabajo a efectos de la aplicación del artículo 10.1 de la LOLS?

Ciertamente, la definición de centro de trabajo se halla formalmente en el artículo 1.5 del TRLET, pero esta caracterización se ha visto sobrepasada por los cambios económicos y productivos que ha sufrido el tejido empresarial español. Tal precepto considera centro de trabajo aquellas unidades productivas con organización específica, sometiéndose su reconocimiento a que sean dadas de alta ante la autoridad laboral, si bien su exigencia normativa ha sido considerada judicialmente como una condición no 
constitutiva, pero de una cierta eficacia probatoria ${ }^{15}$, y definida doctrinalmente como un elemento formal no constitutivo que funciona a modo de presunción iuris tantum ${ }^{16}$. De esta manera, nos encontramos ante un concepto que pone el foco exclusivamente en la autonomía desde el punto de vista técnico ${ }^{17}$, y que ha sido calificada acertadamente por la doctrina como obsoleta ${ }^{18}$, y poco satisfactoria ${ }^{19}$, esencialmente por la falta de adaptación a las estructuras empresariales.

Sin embargo, el mayor problema que presenta el planteamiento del legislador ha sido la falta de concreción de la caracterización de centro de trabajo en las distintas parcelas jurídicas en las que interviene. Parece razonable definir el concepto de trabajo mediante una caracterización no excesivamente detallada, si bien la definición del artículo 1.5 del TRLET es especialmente pobre al no especificar el significado de "organización específica", a fin de tener un marco de referencia general para su interpretación. Ahora bien, la construcción del ejercicio de derechos individuales y colectivos sobre un punto de referencia normativo como es el centro de trabajo hubiera exigido ir un paso más allá, y concretar o matizar el significado de centro de trabajo en función del alcance o características del derecho en cuestión. Y ello debiera ser así si consideramos la distinta relevancia que el concepto "centro de trabajo" puede desempeñar en los distintos escenarios legales $^{20}$.

Contextualizando esta cuestión en el ámbito de las empresas dispersas, y a tenor de una lectura coherente la doctrina jurisprudencial examinada previamente, se plantean dos niveles de discusión claramente diferenciados: aquellas empresas dispersas con centros de trabajo que encajan con la definición legal de centro de trabajo, y aquellas que no ostentan la autonomía suficiente para ser considerados centros de trabajo, entre los que se incluyen microcentros de trabajo y puestos de trabajo dispersos.

\footnotetext{
${ }^{15}$ Vid. Lousada Arochena, J. F., "El centro de trabajo", Revista General de Derecho del Trabajo y de la Seguridad Social, 27, 2011, p. 177 y 178.

${ }^{16}$ Vid. CRUZ Villalón, J., La representación de los trabajadores en la empresa y en el grupo. Un marco legal insuficiente, Editorial Trotta, Valladolid, 1992, p. 32.

${ }^{17}$ Ibid., p. 33.

${ }^{18}$ Vid. CABEZA Pereiro, J., Las elecciones sindicales, Bomarzo, Albacete, 2009, p. 23, que atribuye la desactualización del concepto de centro de trabajo al aumento del grado de autonomía del que disponen los trabajadores en la actualidad, la mejora de las cualificaciones y el impacto de nuevos modelos productivos, que son factores que se suman a un proceso de desaparición de la realidad física del centro de trabajo.

${ }^{19}$ Vid. CRUZ Villalón, J., La representación de los trabajadores en la empresa y en el grupo. Un marco legal insuficiente, cit., p. 35.

${ }^{20}$ Así, por ejemplo, el concepto "centro de trabajo" no ostenta la misma importancia en el establecimiento del sistema de horario flexible en cada centro de trabajo ex artículo 36.1 del TRLET, que en el derecho de permanencia en la empresa con destino a otro centro de trabajo en caso de amortización del puesto de trabajo al amparo del artículo 52.c del TRLET.
} 
Aquellos supuestos en los que la caracterización del centro de trabajo de la empresa dispersa encaja con el concepto legal y jurisprudencial de centro de trabajo no presentan problemas de eficacia. La representación sindical podrá constituirse sin ningún tipo de incidencia, gracias a la doctrina jurisprudencial vigente ${ }^{21}$, que les permite escoger la unidad de adscripción electoral que escoja el propio sindicato.

Sin embargo, los problemas aparecen cuando los puestos de trabajo de las empresas dispersas no encajan con la definición jurídica de centro de trabajo, y, además, no emplean a un gran número de trabajadores. En estos casos, en los que podemos hablar de trabajadores geográficamente dispersos, la definición actual del centro de trabajo $e x$ artículo 1.5 del TRLET se presenta como poco efectiva. El legislador se ha mantenido impasible ante la proliferación de nuevas formas de organización empresariales que en ocasiones incluso eliminan la realidad física del centro de trabajo, planteando problemas de efectividad en el ejercicio del derecho de participación en la empresa. La única opción para constituir la representación sindical de estos trabajadores pasaría por asumir la empresa como unidad de adscripción electoral, vaciando de contenido la opción sindical de escoger cómo instrumentar la constitución de su representación. Tal circunstancia tiene una afectación enorme a la calidad de la representación, y por ende al derecho de participación de los trabajadores en la empresa ex artículo 129.2 CE, ya que impide que las diferentes necesidades y opiniones de cada lugar en el que los trabajadores presentan servicios puedan ser consideradas. La flexibilidad que ofrece el artículo 10.1 de la LOLS desaparece ante el trabajo en empresas dispersas, dada la falta de previsión del legislador de este tipo de fenómenos, produciéndose una curiosa paradoja: la centralización de la representación sindical en estructuras descentralizadas productivamente.

Así pues, ¿podría el sindicato optar por establecer un sistema de representación basado en unidades de adscripción con significado distinto al establecido legalmente? La discusión se centra, en otras palabras, en la posibilidad de moldear por las partes el concepto legal de "centro de trabajo". Distintos argumentos apuntan a ofrecer una respuesta afirmativa a esta cuestión. En primer lugar, debe señalarse el encaje legal de esta posibilidad desde el punto de vista del control de legalidad de las elecciones a los órganos de representación de los trabajadores, tal y como ya señaló la doctrina en su momento $^{22}$. En efecto, el Real Decreto 1844/1994, de 9 de septiembre, por el que se

\footnotetext{
${ }^{21}$ Véase la sentencia del Tribunal Supremo de 18 de julio de 2014 (Rec. 91/2013).

${ }^{22}$ Esta cuestión ya fue indicada por ESCUDERO RODRíGUEZ, R., La representatividad de los sindicatos en el modelo laboral español, Tecnos, Madrid, 1990, p. 120, que, si bien se refería al Real Decreto 1311/1986, de 13 de julio sobre normas para la celebración de elecciones a los órganos de representación de los trabajadores en la Empresa, sigue plenamente vigente a día de hoy.
} 
aprueba el Reglamento de elecciones a órganos de representación de los trabajadores en la empresa, no contempla entre las causas previstas para interponer reclamaciones en materia electoral (artículo 29) que la defectuosa constitución de la unidad electoral anule el proceso de elección a órganos de representación sindical de los trabajadores.

A este primer argumento debe sumársele un segundo elemento capital, y es la expansiva apertura de la jurisprudencia, que permite admitir esta posibilidad. En particular, una lectura coherente con la doctrina jurisprudencial vigente en el marco de la interpretación del artículo 10 de la LOLS, y que en esencia pretende ofrecer una aproximación más efectiva en el ejercicio del derecho a la participación de los trabajadores en la empresa, supondría que las partes también pudieran disponer del concepto "centro de trabajo", ya que resulta contradictorio que los trabajadores sindicados puedan elegir entre empresa o centro de trabajo si la constitución del centro de trabajo recae exclusivamente en la libre organización que considere oportuna el empresario. Y así ha sido reconocido por el Tribunal Supremo en su sentencia de 14 de julio de 2016 (Rec. 161/2015). Este pronunciamiento, que ha pasado ciertamente inadvertido, se encarga de analizar cuál debe de ser el procedimiento adecuado para encauzar una reclamación sobre la circunscripción electoral en el proceso de elección de los representantes sindicales de los trabajadores, pero contiene un razonamiento que abre la puerta a que las partes puedan manipular el concepto "centro de trabajo". Concretamente, la Sala de lo Social atribuye a los promotores del proceso electoral la potestad de definir la unidad electoral sobre la que se quiere delimitar la representación sindical de los trabajadores, en virtud del artículo 67.1 párrafo segundo del TRLET ${ }^{23}$.

De esta manera, sería posible que al inicio del proceso electoral se pactase la unidad de adscripción electoral, que puede ser elegida por los sindicatos entre la empresa o centro de trabajo, y caracterizar las unidades productivas que actuarán como centros de trabajo de forma distinta a la configuración legal del artículo 1.5 del TRLET, pero únicamente a efectos de escoger a la representación sindical de los trabajadores, ex artículo 67.1 párrafo segundo del TRLET. Esta posibilidad adquiere una importancia capital en el caso de las empresas dispersas, ya que permite optimizar la elección de la unidad electoral de la representación sindical, sobre todo si tenemos en cuenta que actualmente nos encontramos en una fase muy temprana de dispersión geográfica del trabajo, y que la tendencia a medio y largo plazo es que la presencia de empresas dispersas aumente, y

\footnotetext{
${ }^{23}$ Véase la STS de 14 de julio de 2016 (Rec. 161/2015), F.D. I, que señala: "5. Y es que el centro de trabajo de la empresa precisa de ser identificado a los efectos de llevar a cabo el proceso de elección de los representantes legales y ello supone que los promotores del proceso electoral habrán de definir esa unidad sobre la que se quiere delimitar la representación, con arreglo a lo dispuesto en los artículos 62 y 63 ET. Así se indica de modo expreso en el artículo 67.1 párrafo segundo [...]".
} 
por lo tanto, la prestación de servicios de trabajadores geográficamente dispersos no adscritos al concepto clásico de centro de trabajo será una dinámica habitual.

No obstante, esta doctrina tiene ciertas limitaciones que son especialmente lesivas para el derecho de los trabajadores a la participación en la empresa. En efecto, es doctrina pacífica del Tribunal Supremo la imposibilidad de agrupar centros de trabajo con menos de seis empleados ${ }^{24}$, que pretende evitar que los sindicatos promotores despojen a los trabajadores de los centros que ocupan entre 6 y 10 trabajadores de la facultad de ser ellos los únicos que pueden decidir, por mayoría, si celebran o no elecciones. La aplicación de esta tesis a la cuestión discutida en esta contribución nos lleva a señalar que la potestad de caracterizar el concepto de centro de trabajo como unidad de adscripción electoral sólo permitiría manipular a las partes la calificación de una unidad productiva como centro de trabajo cuando aquella no cumpla con los requisitos previstos en el artículo 1.5 del TRLET, esto es, unidad productiva, organización específica y alta ante la autoridad laboral, ya que el alcance del concepto debe analizarse en relación con la concreta institución a la que se pretende aplicar en virtud de los múltiples pronunciamientos que ha emitido el Tribunal de Justicia de la Unión Europea sobre el concepto "centro de trabajo" 25 . Sin embargo, el legislador ha dejado espacio para salvar este obstáculo, mediante la aplicación del artículo 8.1.a de la LOLS. Este artículo establece la posibilidad de que los trabajadores afiliados a un sindicato podrán constituir secciones sindicales en el ámbito de la empresa o centro de trabajo, operando como único límite el contenido de los Estatutos del Sindicato. Ello se instrumenta desde un punto de vista absolutamente voluntario, condicionando al contenido que establezca el propio sindicato, dado que el mencionado precepto incluye la expresión "podrán". Y es precisamente en la libertad que confiere a los sindicatos donde radica la posibilidad de evadir la imposibilidad de agrupar centros de trabajo con menos de seis empleados, ya que no se constata ninguna legal respecto al número mínimo de trabajadores. De esta manera, se permite que los afiliados que sean considerados trabajadores geográficamente dispersos puedan tener representación,

\footnotetext{
${ }^{24}$ El mayor exponente de esta doctrina es la Sentencia del Tribunal Supremo de 20 de febrero de 2008 (Rec. 177/2007), que, si bien se refería a la imposibilidad de agrupar centros de trabajos regidos por el artículo 62 del Estatuto de los Trabajadores para la elección de delegados de personal y comités de empresa, es totalmente extrapolable al ámbito de la representación sindical. De modo más reciente, véase la Sentencia del Tribunal Supremo de 14 de julio de 2011 (Rec. 140/2010) y de 11 de febrero de 2015 (Rec. 2872/2013).

${ }^{25}$ Sirvan como ejemplo la STJUE de 30 de abril de 2015, USDAW y Wilson, C-80/14, ECLI:EU:C:2015:291 y la STJUE de 13 de mayo de 2015, Rabal Cañas, C-392/13, ECLI:EU:C:2015:318, que se encargan de precisar la caracterización del centro de trabajo en el marco de los despidos colectivos; o la STJUE de 10 de septiembre de 2015, Federación de Servicios Privados del sindicato CC.OO, C-266/14, ECLI: EU:C:2015:578, que delimita la caracterización de centro de trabajo a los efectos de tiempo de trabajo.
} 
maximizando el ejercicio del derecho de los trabajadores a la participación en la empresa.

\section{Algunas medidas para mejorar la eficacia de la constitución de la representación sindical en empresas dispersas}

La ausencia de un planteamiento amplio y garantista ante estas circunstancias puede derivar en un hecho ciertamente desolador: que los trabajadores de empresas dispersas no puedan ejercer sus derechos de representación colectiva. Si bien se han ofrecido argumentos que permiten afirmar la posibilidad de que adaptar la unidad de adscripción electoral en el marco de las elecciones a la representación sindical, sería conveniente reafirmar esta posibilidad mediante la introducción de algunas modificaciones normativas. $\mathrm{Y}$ a tal efecto, esta contribución concreta a continuación tres propuestas acumulables, a saber, la necesaria ampliación de las unidades electorales a efectos de constitución de las secciones sindicales del artículo 10 de la LOLS, La modificación del concepto legal de centro de trabajo y la actualización de la regulación del comité de empresa conjunto, y el fomento de la intervención de la negociación colectiva.

En primer lugar, sería necesario que el legislador ampliase las unidades de adscripción territorial constatadas en el marco del artículo 10 de la LOLS, incluyendo la expresión "lugares de trabajo", ofreciendo una solución a medida para las empresas dispersas y evitando así una reforma integral del concepto "centro de trabajo". La pretensión de esta propuesta de modificación normativa, que se limita única y exclusivamente a las empresas dispersas, es ofrecer un encaje legal a aquellos trabajadores geográficamente dispersos, que no se hallan adscritos a un centro de trabajo en los términos exigidos legalmente y jurisprudencialmente. La exigencia de una organización productiva autónoma para permitir la elección de representantes sindicales es una premisa que no tiene sentido en el tejido empresarial actual, en el que la realidad física del centro de trabajo está desapareciendo ${ }^{26}$, como consecuencia de la digitalización empresarial. Este fenómeno da paso a una deslocalización de los trabajadores que impide el ejercicio del derecho a la participación colectiva en la empresa.

A diferencia del concepto "centro de trabajo", la expresión "lugar de trabajo" presenta menos condicionantes con relación a cómo desarrolla el empresario la actividad económica. En efecto, la importancia recae en la propia prestación de servicios, garantizando así una conexión más cercana con la génesis del derecho de los trabajadores a la participación en la empresa, que les corresponde por ser trabajadores y no por prestar servicios en una determinada unidad productiva, cuya organización y estructura dependerá exclusivamente del empresario. A sensu contrario, mantener la

${ }^{26}$ Vid. CABEZA PEREIRO, J., Las elecciones sindicales, op. cit., p. 23. 
perspectiva actual, que limita las unidades de adscripción electoral a la empresa o centro de trabajo, condiciona el ejercicio del derecho a la participación en la empresa de los trabajadores a la organización que adopte el propio empresario, y supone que este pueda obstaculizar unilateralmente, ya sea de forma voluntaria o involuntaria, el derecho mencionado.

Todo ello pone de manifiesto una discordancia entre la realidad y la regulación de esta cuestión, y a fin de garantizar un desarrollo efectivo en la diversidad de empresas dispersas, sería recomendable que se optase por una redacción amplia en el marco del artículo 10.1 de la LOLS, con el contenido siguiente: "En las empresas, centros de trabajo, o cualquier agrupación de lugares de trabajo en el caso de empresas dispersas, que ocupen a más de 250 trabajadores". De esta manera, se consigue garantizar que exista representación sindical en aquellas empresas en las que los trabajadores prestan servicios de forma geográficamente dispersa, sin que puedan considerarse centros de trabajo conforme al artículo 1.5 del TRLET.

Otra propuesta para garantizar la presencia de representación sindical en las empresas dispersas pasa por reformar el contenido de la definición legal de centro de trabajo, prevista en el artículo 1.5 TRLET. Cómo ya se ha apuntado anteriormente, la regulación actual del concepto de centro de trabajo es poco efectiva en el marco de las empresas dispersas, debido a la progresiva desaparición de la realidad física de las empresas.

Esta propuesta parece la más idónea en términos de eficiencia, ya que, a diferencia de la anterior, no implicaría introducir nuevos conceptos en el marco de la LOLS, sino mantener el redactado de su artículo 10 y proceder a la actualización del artículo 1.5 del TRLET. En este sentido, las opciones para reformar el concepto de centro de trabajo son diversas, y van desde una modificación integral que mantenga la misma caracterización para los distintos ámbitos en los que se toma en consideración el concepto "centro de trabajo", que es el planteamiento vigente, o bien la creación de nuevas acepciones en determinadas áreas que minimicen el impacto que supondría actualizar esta institución. Así, la propuesta por la que nos inclinamos en esta contribución es la segunda de las opciones manifestadas, ya que supondría introducir una modificación que sólo afectaría en el ámbito de la adscripción electoral de la representación sindical, circunstancia esta que facilitaría enormemente su implementación.

Concretamente, sería recomendable que, a efectos de considerar la existencia de uno o varios centros de trabajo como unidades de adscripción electoral en las empresas dispersas, se constatase en el marco del artículo 1.5 del TRLET una definición explícita y diferenciada del concepto "centro de trabajo", que omitiese cualquier referencia a la existencia de una organización específica y al requisito de dar de alta ante la autoridad 
laboral. La razón de su supresión radica en que ambas condiciones dependen ciertamente de la voluntad del empresario, que es el responsable de organizar los medios de producción y el obligado legal a la comunicación de la apertura de los centros de trabajo.

Y llegados a este punto, ¿qué elementos debería integrar su definición como unidad de adscripción electoral? Esencialmente, debería incluir la referencia al conjunto de puestos de trabajo que se integran en el desarrollo de la actividad económica de una empresa. Además, sería recomendable añadir una mención expresa a la consideración como centros de trabajo de aquellos puestos de trabajo geográficamente deslocalizados, a fin de que los trabajadores puedan seguir manteniendo el derecho de participación en la empresa. En este sentido, hay distintos ejemplos en nuestro ordenamiento jurídico, que, aplicados a las empresas dispersas, ofrecerían un ejercicio más efectivo del derecho de los trabajadores a la participación en la empresa. Así, por ejemplo, el artículo 2.a del Real Decreto 171/2004, de 30 de enero, por el que se desarrolla el artículo 24 de la Ley 31/1995, de 8 de noviembre, de Prevención de Riesgos Laborales, en materia de coordinación de actividades empresariales (en adelante, RD 171/2004), define centro de trabajo como cualquier área, edificada o no, en la que los trabajadores deban permanecer o a la que deban acceder por razón de su trabajo. Pero este no ha sido el único caso que se ha apartado de la caracterización prevista en el artículo 1.5 del TRLET. La disposición adicional tercera del Real Decreto 1844/1994, de 9 de septiembre, por el que se aprueba el Reglamento de elecciones a órganos de representación de los trabajadores en la empresa (en adelante, RD 1844/1994) se ha encargado de exigir la agrupación de los establecimientos dependientes u organismos que radiquen en una misma provincia como un único centro de trabajo, requiriendo para ello que se encuentren incluidos en el ámbito de aplicación de un mismo Convenio Colectivo. Como puede observarse, el legislador ha previsto otras formas de definir el significado de centro de trabajo, que, aplicadas a efectos de determinar la unidad de adscripción electoral en el marco de las elecciones a representantes sindicales, serían más eficaces que la prevista en el mencionado artículo 1.5 del TRLET.

Así, con la eliminación del requisito de organización específica y tramitación del alta ante la autoridad laboral, y la reformulación del concepto de centro de trabajo hacia una definición en la que el empresario no puede intervenir en su conceptualización, introduciendo por ejemplo la posibilidad de considerar centro de trabajo aquellos puestos o lugares de trabajo a los que trabajador deba acceder o permanecer para prestar sus servicios y/o que aquellos se encuentren incluidos en el ámbito de aplicación de un mismo Convenio Colectivo, permitiría ofrecer un concepto de centro de trabajo mucho más actualizado y adaptado a su finalidad. 
Sin embargo, esta segunda propuesta también debería incluir la integración de una figura existente en la representación unitaria de los trabajadores, a fin de reforzar la constitución de la representación sindical en las empresas dispersas, pero introduciendo algunas modificaciones en su régimen jurídico. Nos estamos refiriendo al comité conjunto, previsto en el artículo 63.2 del TRLET como un instrumento de representación de trabajadores, que actúa como una extensión preceptiva ${ }^{27}$, para aquellos centros de trabajo cuyos censos no alcancen los cincuenta trabajadores, pero que en su conjunto lo sumen en un determinado ámbito territorial ${ }^{28}$. Esta institución parece especialmente indicada para las empresas dispersas, toda vez que favorece que una pluralidad de pequeños centros de trabajo pueda constituir un órgano de representación sindical, pero su previsión sólo ha sido legalmente constatada para la representación unitaria. Si bien es cierto que la constitución de la representación sindical no está sometida a las mismas reglas que las relativas a la creación de la representación unitaria, gracias a la distinción jurisprudencial de los representantes sindicales ad extra/ad intra, sería conveniente trasladar, con algunas modificaciones, la figura del comité conjunto a la representación sindical en las empresas dispersas. Las razones que así lo justifican se fundamentan en la reducción de facultades de los representantes sindicales que no alcanzan el umbral mínimo establecido en el artículo 10.1 de la LOLS, y la necesidad de representación en estos casos, en los que nos encontramos mayoritariamente ante puestos de trabajo dispersos.

En particular, sería recomendable el reconocimiento legal de las secciones sindicales conjuntas, que permitiera la suma de centros de trabajo con una plantilla inferior a 250 trabajadores, incluyendo aquellos centros de trabajo con menos de 6 trabajadores, que se suma a otras propuestas que se han realizado por la doctrina científica ${ }^{29}$. Es cierto que esta posibilidad ya puede llevarse a cabo gracias al poder de autoorganización del propio sindicato ex artículo 8 de la $\operatorname{LOLS}^{30}$, pero esta opción no supone el

\footnotetext{
${ }^{27}$ Véase la STS de 20 de febrero de 2008 (Rec. 77/2007), entre otras. Para un examen en profundidad de las implicaciones que tuvo esta sentencia, véase Cabeza Pereiro, J., "Al paso de la jurisprudencia del TS relativa a las unidades electorales después de la S de 20 febrero 2008", Relaciones Laborales, $\mathrm{n}^{\circ}$. 23-24, 2008.

${ }^{28} \mathrm{El}$ artículo 63.2 del TRLET delimita el ámbito de aplicación a que la empresa tenga dos o más centros de trabajo inferiores a cincuenta trabajadores en la misma provincia, o en municipios limítrofes.

${ }^{29}$ Así, por ejemplo, véase YSAS MOLINERo, H., "Reflexiones sobre el comité conjunto o cómo reestructurar los órganos unitarios para mejorar la cobertura representativa en las empresas" en V.V.A.A, Descentralización productiva: nuevas formas de trabajo y organización empresarial, ediciones cinca, Madrid, 2018, (formato electrónico); NIETO ROJAS, P., La representación de los trabajadores en la empresa, Lex Nova, Cizur Menor, 2015, p. 189-201, entre otros.

${ }^{30}$ Vid. Merino Segovia, A., "La acción sindical en la empresa. Nuevas orientaciones en la doctrina judicial a propósito del crédito horario del delegado sindical", Revista de Derecho Social, nº 70, 2015, p. 181-182.
} 
reconocimiento de los derechos previstos en el artículo 8.2 de la LOLS, salvo que el convenio colectivo de aplicación así lo constate. Tal circunstancia podría ser aceptable en el supuesto de empresas no dispersas, pero no en el caso de aquellos empleadores con microcentros de trabajo dispersos, ya que a la desconexión propia como consecuencia de la distancia entre puestos de trabajo se le suma el factor de la reducida plantilla por cada unidad de producción. Y ante este escenario, sería conveniente garantizar que estos trabajadores puedan constituir la representación sindical con el máximo de las garantías y derechos previstos normativamente, especialmente si tenemos en cuenta las circunstancias en las que se lleva a cabo la actividad económica en este tipo de empresas.

Por último, es necesario destacar el importantísimo papel que debe desempeñar la negociación colectiva potenciando la representación sindical en las empresas dispersas. La flexibilidad exigida para adaptar la forma en la que se constituye esta vía de representación de los trabajadores en el marco de los distintos sectores económicos sitúa a la negociación colectiva como un agente idóneo para su potenciación. De hecho, el legislador es consciente de su potencial, e instrumentó un marco jurídico, previsto en la LOLS, con multitud de espacios en los que puede intervenir la negociación colectiva. Así, por ejemplo, los artículos 8.2, 10.2 y 10.3 de la LOLS permiten que los convenios colectivos amplíen el número de delegados sindicales que corresponde en función de una determinada plantilla y/o establezcan nuevos derechos tanto para la representación unitaria como sindical. Ante este escenario, convendría fomentar la creación de disposiciones convencionales específicas que potencien la constitución de la representación sindical, a través de la mejora habilitada a tal efecto por la LOLS, para esta nueva realidad empresarial en la que la dispersión de los puestos de trabajo es la tónica habitual. En términos prácticos, ello comprendería intervenciones ya reconocidas por la jurisprudencia, como por ejemplo rebajar de 250 a 150 el número exigido de trabajadores a efectos de determinar el número de delegados sindicales ${ }^{31}$.

Para un estudio en profundidad de esta figura, véase RoDRíGUEZ-PIÑERO, M., "El reconocimiento legal de los delegados sindicales y las representaciones sindicales al margen de la ley", Relaciones laborales, $\mathrm{n}^{\circ}$ 14, 1989; AHUMADA VilLaLba, B., "Secciones sindicales intercentros y delegados sindicales", Revista Española de Derecho del Trabajo, $\mathrm{n}^{\circ}$ 43, 1990, p. 535-540; SAEZ LARA, C., Representación y acción sindical en la empresa, Ministerio de Trabajo y Seguridad Social, Madrid, 1992, p. 168-170; VAldeOlivas García, Y., Antisindicalidad y relaciones de trabajo (Un estudio de la conducta antisindical en la empresa), Civitas, Madrid, 1994, p. 245-246..

${ }^{31}$ Véase la STS de 17 de junio de 2014 (Rec. 157/2013), que sostiene que "[...] en la expresión legal "ampliar el número de delegados" se puede considerar comprendida la situación fáctica del caso de autos: al rebajar de 250 a 150 el número mínimo exigido, se permite tener un delegado donde antes se tendría cero". 
Es cierto que el fomento y mejora de esta vía de representación en la empresa exige la afiliación a un sindicato, que es el pilar esencial en el que se sustenta ex artículo 8.1 de la LOLS, pero este es precisamente un fenómeno que generaría una necesaria consecuencia positiva en el ordenamiento jurídico español: el crecimiento de la acción sindical. En efecto, la acción sindical está sufriendo una crisis de gran entidad ante una realidad empresarial que apuesta por la descentralización, en la que impera la desconexión entre trabajadores/as por hallarse en centros de trabajo geográficamente distantes. Tal circunstancia dificulta la posibilidad de constituir la representación sindical de los trabajadores. Y aunque pueda parecer una paradoja, el establecimiento de nuevas formas de organización empresarial basadas en la economía colaborativa puede devolver la importancia que han ido perdiendo los sindicatos a lo largo de los últimos años, ya que se presenta como una vía flexible y efectiva para la representación efectiva de los trabajadores.

\section{Bibliografía}

Ahumada Villalba, B., "Secciones sindicales intercentros y delegados sindicales", Revista Española de Derecho del Trabajo, núm. 43, 1990.

Alós, R., Beneyto, P. J., Jódar, P., Molina, O. y Vidal, S., La representación sindical en España, Fundación $1^{\circ}$ de Mayo, 2015.

BAylos GraU, A., "La «huída» del Derecho del trabajo. Tendencias y límites de la deslaboralización.", en El trabajo ante el cambio de siglo: un tratamiento multidisciplinar, Marcial Pons, Barcelona, 2000.

CABeza Pereiro, J., Las elecciones sindicales, Bomarzo, Albacete, 2009.

Cabeza Pereiro, J., "Al paso de la jurisprudencia del TS relativa a las unidades electorales después de la S de 20 febrero 2008”, Relaciones Laborales, n 23-24, 2008

Casas BaAmonde, M. E. y Escudero Rodríguez, R., "Representación sindical en el sistema español de relaciones laborales", Revista española de Derecho del Trabajo, vol. $17,1984$.

CRUZ VILlalón, J., La representación de los trabajadores en la empresa y en el grupo. Un marco legal insuficiente, Editorial Trotta, Valladolid, 1992.

ESCUDERO RODRÍGUEZ, R., La representatividad de los sindicatos en el modelo laboral español, Tecnos, Madrid, 1990. 
Lousada Arochena, J. F., "El centro de trabajo", Revista General de Derecho del Trabajo y de la Seguridad Social, n 27, 2011.

Merino Segovia, A., "La acción sindical en la empresa. Nuevas orientaciones en la doctrina judicial a propósito del crédito horario del delegado sindical", Revista de Derecho Social, $\mathrm{n}^{\mathrm{0}}$ 70, 2015.

MiÑARRO YANINI, M. "La representación sindical se "independiza" de la unitaria: el crédito horario de los delegados sindicales (Comentario a la Sentencia del Tribunal Supremo, Sala 4. ${ }^{\text {, }}$ de 18 de julio de 2014, rec. núm. 91/2013)", Revista de trabajo y Seguridad Social del Centro de Estudios Financieros, n 381, 2014.

Moll NoguerA, R., "Crédito de horas retribuidas de los delegados sindicales: (Sentencia del Tribunal Supremo de 18 de julio de 2014, RC 91/2013)", Revista de información laboral, n 11, 2014.

Nieto Rojas, P., La representación de los trabajadores en la empresa, Lex Nova. Cizur Menor, 2015

PAdilla MelÉndez, A.; SeRARols I TARRÉS, C., "Las caractertiscas del empresario y el éxito de las empresas puramente digitales", Información Comercial Española, ICE: Revista de economía, vol. 833, 2006.

PÁRAmo Montero, P., "Las nuevas formas emergentes de trabajo. Especial referencia a la economía colaborativa", Revista del Ministerio de Empleo y Seguridad Social, vol. $128,2017$.

RodrígueZ-PIÑERO, M., "El reconocimiento legal de los delegados sindicales y las representaciones sindicales al margen de la ley", Relaciones laborales, n 14, 1989.

SAez LARA, C., Representación y acción sindical en la empresa, Ministerio de Trabajo y Seguridad Social, Madrid, 1992.

SAÉZ LARA, C., "La representación colectiva de los trabajadores en la empresa", Revista del Ministerio de Trabajo y Asuntos Sociales, $\mathrm{n}^{\circ}$ 58, 2005.

Todolí Signes, A., "La regulación especial del trabajo en la Gig Economy", Redes, vol. $15,2017$. 
Ysas Molinero, H., "Reflexiones sobre el comité conjunto o cómo reestructurar los órganos unitarios para mejorar la cobertura representativa en las empresas" en V.V.A.A, Descentralización productiva: nuevas formas de trabajo y organización empresarial, ediciones cinca, Madrid, 2018, (formato electrónico).

VALDÉS DAL-Re, F. y Molero MARAÑON, L., "Presentación”, en La representación de los trabajadores en las nuevas organizaciones de empresa, Ministerio de Trabajo e Inmigración, Madrid, 2010.

Valdeolivas García, Y., Antisindicalidad y relaciones de trabajo (Un estudio de la conducta antisindical en la empresa), Civitas, Madrid, 1994. 